\title{
Temporal evolution of the domain structure in a Poisson-Voronoi nucleation and growth transformation. Results for one and three dimensions
}

\author{
Eloi Pineda* \\ Departament de Física i Enginyeria Nuclear, \\ ESAB, Universitat Politècnica de Catalunya \\ Avinguda del Canal Oímpic 15, 08860 - Castelldefels, SPAIN \\ Daniel Crespo \\ Departament de Física Aplicada, EPSC, \\ Universitat Politècnica de Catalunya \\ Avinguda del Canal Oímpic 15, 08860 - Castelldefels, SPAIN
}

(Dated: May 30, 2008)

\begin{abstract}
The distribution of spatial domain structures originated during one and three dimensional Poisson-Voronoi transformations are computed analytically extending the recently obtained results for the two dimensional case. The presented method gives a full description of the developed microstructure and is valid for tessellations of any dimensionality. The temporal and spatial dependences of the domain structure are completely discriminated and separated, showing the existence of geometric configurations independent of time. A single computation of the probability distribution of these geometric configurations allows us to calculate the total free-boundary and size probability distributions at any desired time. The obtained results show full agreement with stochastic simulations and reproduce completely the previously existing partial results. A discussion about the potential applications of the method to the calculation of other geometrical properties and the characteristics of the final static structure leading to a gamma distribution of sizes is also presented.
\end{abstract}

PACS numbers: 02.50.-r, 02.50.Ey, 05.70.Fh, 81.30-t

*Electronic address: eloi.pineda@upc.edu; URL: http://mie.esab.upc.es/eloi 


\section{INTRODUCTION}

Partition of space in separate domains giving rise to a tessellation is common in many physical systems. In such systems, the geometrical properties of the domain structure determine most of the macroscopic properties and influence the posterior dynamics and evolution of the system. Grain microstructure in metals, cellular structure in granular materials or foams and the domain structure of magnetic materials are some typical examples of these kind of systems. In this work we analyze the domain structure generated during a PoissonVoronoi (PV) nucleation and growth transformation. In a PV transformation, a set of randomly distributed nucleation points starts growing simultaneously and isotropically occupying the untransformed space. The collision of two growing domains defines a static boundary and the two domains remain distinguishable. The initial state of the system is a random point distribution of nucleation sites, while the final stage -when all the untransformed space is occupied- is a Poisson-Voronoi cellular network or tessellation [1][2]. In between, the system consists of new phase domains partly in contact with each other and surrounded by untransformed regions.

In a one-dimensional space, the domains are line segments which progressively occupy the adjacent untransformed space at both sides of the segment. After colliding with other domains at both sides, these mono-dimensional domains remain static. In two and threedimensional spaces, the initial circular or spherical growing domains become progressively transformed into polygonal or polyhedral cells because of the collisions with their neighbours. Figures 1 and 2 show the configuration of a given region at three different stages during a one and a two-dimensional PV transformations respectively. This type of transformation is also referred as transformation with saturation of nucleation sites [3][4][5], growth of pre-existing nuclei [6], cell model [7][8] or crystallization with simultaneous nucleation [9].

A PV transformation is completely determined by two parameters, namely, the intensity of the Poisson process $\rho$, that is the density of initial seeds or nucleation points, and the growth rate of the domains $u$; these parameters determine the kinetics and the domain structure at any time $t$. Choosing the time origin $t=0$ at the beginning of the transformation,

the space occupied by a domain without collisions at time $t$ is $K_{D}(u t)^{D}$, where $D$ is the dimension of the system and

$$
K_{D}=\frac{2 \pi^{\frac{D}{2}}}{D \Gamma(D / 2)}
$$


Then the transformed space fraction $x(t)$ is obtained from

$$
x(t)=1-\exp \left[-\rho K_{D}(u t)^{D}\right]
$$

which is the well-known Avrami or KJMA (Kolmogorov-Johnson and Mehl-Avrami) equation for this kind of transformation [10][11] [12]. The evolution of $x(t)$ for $D=1$ and 2 is also depicted in figures 1 and 2. As all domains have the same growth rate, the spatial configuration at a given value of $x(t)$ is independent of the value of $u$. For the sake of simplicity, in this work we will consider $u=1$; the results for any other value of the growth rate are easily obtained with the appropriate time scaling.

At the end of the transformation, this means when $x(t) \rightarrow 1$ and $t \rightarrow \infty$, the whole space is occupied by static domains forming a PV tessellation, whose geometrical features have been widely studied [2][13][14][15]. For instance, the probability density function (PDF) of domain sizes of a PV tessellation is known to be a Gamma probability function

$$
f(a)=\frac{(\nu \rho)^{\nu}}{\Gamma(\nu)} a^{\nu-1} \exp (-\nu \rho a),
$$

where the size $a$ is defined as the length, area or volume occupied by a domain and the exponent $\nu$ is found to be $2,3.575$ and 5.586 for $D=1,2$ and 3 respectively [16]. These result is analytically deduced for the one-dimensional case [7][17], but it has not been mathematically proofed for $D>1$. For $D>1$, the validity of the Gamma distribution has been checked against stochastic simulations by fitting the numerical values of the exponent $\nu[13][15][16]$.

The temporal evolution of the domain size distribution in a one-dimensional PV transformation was completely described in reference [17]. In a one-dimensional system, the domains can be treated as growing line segments which can be in just three collision states: a) no-collisions, b) collision at one side, c) collisions at each side. This simplicity of the collision process facilitates an analytical treatment that gives a complete description of the domain structure at any time $t$ during the transformation. The PDF of other characteristics such as the length of untransformed gaps or the size of domain aggregates was also derived by Schulze [17].

Finding an explicit solution for the temporal evolution of the domain structure for $D>1$ remains an unsolved problem. There is an intrinsic topological reason for this, the number of growth directions in one-dimensional systems is finite, while it becomes infinite in higher dimensional systems. This prevents the extension of the method used in $D=1$ to higher 
dimensions. Only very recently [18][19] the authors presented an analytical method for calculating the PDF of any geometrical characteristic of the domains at any finite time $t$ during a PV transformation. This was possible by revealing the underlying self-similarity of the growth-and-impingement process along the transformation. By this approach the deduced analytical integral expressions can be calculated numerically to any desired accuracy, and results for the domain size and domain free boundary distributions in a two-dimensional transformation were presented. Furthermore, one of the main theoretical results of such method is the fact that it is fully independent of the dimensionality of the space under consideration. We show that in this paper by presenting a detailed calculation for the cases of $D=1$ and $D=3$.

In section II we recapitulate the method and we generalize them to deal with PV transformations in spaces of any dimension. In section III we present the application of the method to a one-dimensional PV transformation. Although the total size distribution for the $D=1$ case was previously calculated, the new derivation presented here completes the generalization of the method. The explicit results obtained for the one-dimensional system provide additional information of the spatial configuration and they allow us a direct comparison with $D>1$ systems, this being particularly useful in the discussions given in following sections. In section IV we extent the calculation method to $D>1$ and to an arbitrary domain property. Computations of the free boundary and size of the domains are presented in detail for the three-dimensional case, which is a case with high physical significance. Section V is devoted to the final static structure, the potentiality of the presented calculation method to provide a derivation of the Gamma distribution of sizes observed in PV tessellations is discussed. The similarities between the $D=1$ results, from which the Gamma distribution is analytically obtained, and the $D=2$ and 3 computations are presented and discussed. Finally, in section VI we summarize the main results of the work.

\section{TIME-INVARIANT PROBABILITY DISTRIBUTIONS OF GEOMETRIC CONFIGURATIONS}

The method presented in reference [18] is based on the calculation of the time-invariant probabilities of geometric configurations for domains with a certain number of extended collisions. Considering, without loss of generality, $u=1$, the number $k$ of extended collisions 
of a domain with nucleation point $O$ is defined as the number of neighbouring nucleation points $O_{i}$ within a distance $2 t$ of $O$. This is equal to the number of neighbouring domains that would impinge with the domain if there was no screening by previous collisions. Considering a Poisson point-distribution with density $\rho$ in a space of dimension $D$, the probability of a domain to have $k$ extended collisions at time $t$ is given by

$$
T_{k}(t)=\frac{\left(K_{D} 2^{D} t^{D} \rho\right)^{k} \exp \left(-K_{D} 2^{D} t^{D} \rho\right)}{k !},
$$

which is the probability of finding exactly $k$ points within a region of volume $K_{D}(2 t)^{D}$. At $t=0$ all the domains have $k=0$ collisions, then $T_{0}(0)=1$ and $T_{k}(0)=0$ for any $k>0$. As the transformation proceeds, each $T_{k}(t)$ function increases until reaching a maximum value and then diminishes towards zero. A graphical representation of the $T_{k}(t)$ functions for $D=2$ was given in reference [19].

Following the procedure detailed in references [18] and [19], the collision configuration of a domain can be defined by the positions $\left\{O_{i}\right\}(i=1 . . k)$ of the $k$ surrounding nucleation points nearer than a distance $2 t$. In a $D=1$ system these positions are determined just by the distances $\pm 2 t_{i}$ between $O_{i}$ and $O$, where $t_{i}$ is the collision time between the two domains. In $D=2$ and 3 spaces these positions can be expressed as $O_{i}=\left(2 t_{i}, \theta_{i}\right)$ and $O_{i}=\left(2 t_{i}, \theta_{i}, \varphi_{i}\right)$ in polar coordinates centered at the domain origin. Using a normalization $l_{i}=t_{i} / t$ of the collision times, the collision configuration of a certain domain can be defined by the set $\left\{l_{i}\right\}$, $\left\{l_{i}, \theta_{i}\right\}$ or $\left\{l_{i}, \theta_{i}, \varphi_{i}\right\}$ for $D=1,2$ or 3 respectively. The probability of finding a domain with a certain collision configuration at time $t$ is found multiplying the probability $\rho d V_{D i}$ of finding a nucleation point within $d V_{D i}$ and the probability $\exp \left(-K_{D} 2^{D} t^{D} \rho\right)$ of finding no other nucleation point within the $K_{D}(2 t)^{D}$ region, this probability can be written as

$$
P_{k}\left(O_{1}, \ldots, O_{k}, t\right)=T_{k}(t) \frac{k !}{K_{D}} \prod_{i=1}^{k} d V_{D i},
$$

where $d V_{D i}$ is the volume differential element at position $O_{i}$. For the cases of $D=1, D=2$ and $D=3$ we have $d V_{1 i}=2 d l_{i}, d V_{2 i}=l_{i} d l_{i} d \theta_{i}$ and $d V_{3 i}=l_{i}^{2} \sin \left(\theta_{i}\right) d l_{i} d \theta_{i} d \varphi_{i}$. The essential point here comes from the fact that the probability of equation (5) is composed by two factors, one only dependent on time and the other only dependent on the collision configuration. This result implies that the evolution of the domain structure in a PV transformation can be interpreted as a sum of domain $k$-populations with time invariant normalized geometric properties, each one of this populations containing a fraction of domains at time $t$ given 
by equation (4). In the above expression we implicitly assumed a temporal order for the collision times, that is $l_{i-1}<l_{i}$. If this temporal order above chosen is not considered the right hand side (rhs) of equation (5) should be divided by $k$ !.

If the probability of finding a specific geometrical configuration among the domains with given $k$ is time invariant, then the PDF of any geometrical property of these domains must be also time invariant. For a certain geometric characteristic, the calculation of these timeinvariant functions will allow us the construction of the overall PDF at any time $t$ just by adding the contributions $T_{k}(t)$ of each $k$-population. In the following sections this is carried out for the free-boundary and for the size of the domains. Finally, the explicit expressions for the probability of a certain collision configuration of a domain with $k$ extended collisions, as they will be used in the following sections, can be written as

$$
\begin{aligned}
k ! \prod_{i=1}^{k} d l_{i}, & \text { for } D & =1 \\
\frac{k !}{\pi^{k}} \prod_{i=1}^{k} l_{i} d l_{i} d \theta_{i}, & \text { for } D & =2 \\
k !\left(\frac{3}{4 \pi}\right)^{k} \prod_{i=1}^{k} l_{i}^{2} \sin \left(\theta_{i}\right) d l_{i} d \theta_{i} d \varphi_{i}, & \text { for } D & =3
\end{aligned}
$$

which correspond to the time-invariant term in the rhs of equation (5) for the specific cases of one, two and three dimensions.

\section{RESULTS FOR $\mathrm{D}=1$}

\section{A. Temporal evolution of the free boundary distribution}

The free boundary fraction $b$ of a domain is defined as the fraction of the domain boundary in contact with untransformed space. In the case of a $D=1 \mathrm{PV}$ transformation, the free boundary fraction becomes a discrete variable having only three possible values: $b=1$ (domains without collisions), $b=1 / 2$ (domains with collisions in one side) and $b=0$

(domains with collisions in each side). Thanks to this simplicity, the probability $Q_{k, b}$ of being in one of these three cases for a domain with $k$ extended collisions can be easily calculated.

For $k=0$ and $k=1$ all the domains have $b=1$ and $b=1 / 2$ respectiveley, and so

$$
Q_{0,1}=1, \quad Q_{0, \frac{1}{2}}=0, \quad Q_{0,0}=0
$$


and

$$
Q_{1,1}=0, \quad Q_{1, \frac{1}{2}}=1, \quad Q_{1,0}=0 .
$$

After that, each new extended collision has the same probability of being at either side of the domain. The domains with collisions in just one side maintain $b=1 / 2$ while the domains with collisions in both sides have $b=0$. This leads to the following general expression

$$
Q_{k, 1}=0, \quad Q_{k, \frac{1}{2}}=\left(\frac{1}{2}\right)^{k-1}, \quad Q_{k, 0}=1-\left(\frac{1}{2}\right)^{k-1}
$$

for $k>0$. These expressions give the fraction of domains in each of the three possible boundary states for the population of domains with $k$ extended collisions, these probabilities are time invariant as expected. Therefore, the overall probability $Q_{b}(t)$ of finding a domain in one of the three states at a certain time $t$ can be easily obtained adding the contributions of each $k$-population, that is

$$
Q_{b}(t)=\sum_{k=0}^{\infty} Q_{k, b} T_{k}(t) .
$$

Substituting the $T_{k}(t)$ expression of equation (4) in the above equation we obtain

$$
\begin{gathered}
Q_{1}(t)=\exp (-4 \rho t) \\
Q_{\frac{1}{2}}(t)=2[1-\exp (-2 \rho t)] \exp (-2 \rho t) . \\
Q_{0}(t)=[1-\exp (-2 \rho t)]^{2}
\end{gathered}
$$

The previous expressions can be derived using other arguments, for instance they can be expressed in function of the transformed fraction $x(t)=1-\exp (-2 \rho t)$ as

$$
\begin{gathered}
Q_{1}(t)=[1-x(t)]^{2} \\
Q_{\frac{1}{2}}(t)=2[1-x(t)] x(t) \\
Q_{0}(t)=x(t)^{2}
\end{gathered}
$$

where it becomes obvious that $Q_{1}(t), Q_{0}(t)$ and $Q_{\frac{1}{2}}(t)$ are respectively the probability of two randomly chosen points to be found both in untransformed space, both in transformed space and each one in a different state. The simplicity of the collision process in a $D=1$ transformation makes the derivation of the above probabilities possible by many different ways. The derivation in terms of extended collisions is presented here for completeness. 


\section{B. Temporal evolution of the size distribution}

In a $D=1 \mathrm{PV}$ transformation the size of a domain corresponds to its length $a$. We define here the normalized size of a domain as

$$
s=\frac{a}{2 t}
$$

where $2 t$ is the size of a domain without any collision blocking its growth. These size $s$ is completely determined by the particular collision sequence of the domain $\left\{l_{i}\right\}$. Therefore, the time-invariant size $\mathrm{PDF} g_{k}(s)$ for domains with a given $k$ can be calculated using the probability for a certain collision sequence given by equation (6) and integrating over all the possible sequences:

$$
g_{k}(s) d s=k ! \int_{l_{1}=0}^{1} \ldots \int_{l_{k}=l_{k-1}}^{1} \delta\left(s-S_{k}\left(l_{1}, \ldots, l_{k}\right)\right) \prod_{i=1}^{k} d l_{i}
$$

where $\delta($.$) is the Dirac delta function and S_{k}\left(l_{1}, \ldots, l_{k}\right)$ is a function which calculates $s$ for a particular collision sequence $\left\{l_{i}\right\}$.

The function $S_{k}\left(l_{1}, \ldots, l_{k}\right)$ can be explicitly obtained in $D=1$. For domains with $k=0$ and $k=1$ it is obvious that

$$
S_{0}=1, \quad S_{1}\left(l_{1}\right)=\frac{1+l_{1}}{2}
$$

For $k>0$, a discussion similar to the one in the free-boundary calculation leads to

$$
S_{k}\left(l_{1}, \ldots, l_{k}\right)= \begin{cases}\frac{1+l_{1}}{2}, & \text { with probability }\left(\frac{1}{2}\right)^{k-1} \\ \frac{l_{1}+l_{i}}{2}, & \text { with probability }\left(\frac{1}{2}\right)^{i-1}\end{cases}
$$

In order to clarify the origin of the previous expression the derivations for $k=2$ and $k=3$ will be detailed as examples. For a domain with $k=2$ and a certain $\left\{l_{1}, l_{2}\right\}$ with $l_{1}<l_{2}$, there is an equal probability of having the two extended collisions in the same side or in different sides. In the former case the growth is stopped just in one side of the domain at the first collision time, and the normalized domain size is then $S_{2}\left(l_{1}, l_{2}\right)=\frac{1+l_{1}}{2}$. In the latter case the growth is stopped at both sides of the domain and the size is $S_{2}=\frac{l_{1}+l_{2}}{2}$. For $k=3$ and a certain $\left\{l_{1}, l_{2}, l_{3}\right\}$ with $l_{1}<l_{2}<l_{3}$, there is a $1 / 4$ probability of having all the collisions at the same side and so $S_{3}\left(l_{1}, l_{2}, l_{3}\right)=\frac{1+l_{1}}{2}$, there is a $1 / 2$ probability of having 
$l_{1}$ and $l_{2}$ at different sides and so $S_{3}\left(l_{1}, l_{2}, l_{3}\right)=\frac{l_{1}+l_{2}}{2}$, and there is a $1 / 4$ probability of having the first and the second collision at the same side and the third one in the opposite side, this giving $S_{3}\left(l_{1}, l_{2}, l_{3}\right)=\frac{l_{1}+l_{3}}{2}$. Extending this reasoning to larger $k$ numbers the above general expression for $S_{k}\left(l_{1}, \ldots, l_{k}\right)$ is obtained for any $k>0$.

Now, the functions $g_{k}(s)$ can be obtained calculating the integration in equation (14). The first size PDF corresponding to $k=0$ is then

$$
g_{0}(s)=\delta(s-1)
$$

For $k>0$ the substitution of equation (16) in equation (14) leads to

$$
g_{k}(s) d s=k ! \int_{l_{1}=0}^{1} \ldots \int_{l_{k}=l_{k-1}}^{1}\left[\left(\frac{1}{2}\right)^{k-1} \delta\left(s-\frac{1+l_{1}}{2}\right)+\sum_{i=2}^{k}\left(\frac{1}{2}\right)^{i-1} \delta\left(s-\frac{l_{1}+l_{i}}{2}\right)\right] \prod_{i=1}^{k} d l_{i} .
$$

The integration of the previous equation gives

$$
g_{k}(s)=\left\{\begin{array}{c}
k(k-1) s(1-s)^{k-2}, \quad \text { for } s<\frac{1}{2} \\
k(k+1)(1-s)^{k-1}, \quad \text { for } \frac{1}{2}<s<1
\end{array} .\right.
$$

A detailed derivation of this result is given in appendix A.

Figure 3 shows the $g_{k}(s)$ functions for $k=1,2,3,4$ and 5 . As shown in figure 3 the time-invariant size probability density functions are strickingly simple. Using the expressions obtained for the $g_{k}(s)$ functions, the total PDF of normalized sizes can be obtained as

$$
g_{\text {total }}(s, t)=\sum_{k=0}^{\infty} g_{k}(s) T_{k}(t)
$$

where the time evolution of the overall system is obtained as a summation of the timeinvariant functions, weighted by the fraction of domains with certain $k$ at certain time $t$ given by equation (4). Figure 4 shows the $g_{\text {total }}(s, t)$ when the overall transformed fraction is $x(t)=0.6$. At this stage of the transformation the domains with $k=0$ constitute the $16 \%$ of all the domains, and $99 \%$ of the domains have $k \leq 5$. The total size probability density function of figure 4 was computed adding the contributions of the $g_{k}(s)$ distributions with $k$ ranging from 0 to 5 , which are also shown in the figure. The validity of the obtanied results was tested by comparing to stochastic simulations. An unidimensional array of $2^{14}$ positions was progressively covered by approximately 330 seeds randomly spread. Bars in figure 4 correspond to the average of 100 of these simulations, showing full agreement with 
the computed $g_{\text {total }}(s, t)$. It must be noted that the height of the bar at $s=1$, that is the bin corresponding to the domains without collisions, has been artificially reduced in order to improve the appraising of the details of the rest of the distribution. The probability density function at $s=1$ is a Dirac delta function, and so the height of the bar in a histogram depends on the bin size.

The number of $g_{k}(s)$ distributions needed to compute the total PDF depends on the time $t$ and the desired accuracy of the calculation. However, in the case of $D=1$ an explicit result for the total size PDF can be obtained from equation (20). Using equations (4), (17) and (19), the infinite series of equation (20) can be analytically solved. Then, from the normalized size PDF $g_{\text {total }}(s, t)$ the total size PDF $f_{D=1}(a, t)$ is obtained using the variable change in equation (13) giving

$$
\begin{gathered}
f_{D=1}(a, t)=g_{\text {total }}(s, t) \frac{d s}{d a}= \\
=\exp (-4 \rho t) \delta(a-2 t)+\exp (-2 \rho a)\left[2 \rho(2+4 \rho t-2 \rho a) H(a-t)+4 \rho^{2} a H(t-a)\right]
\end{gathered}
$$

where $H($.$) is the Heaviside step function. The first term in the rhs of the equation corre-$ sponds to the PDF of domains with no-collisions, all of them with $a=2 t$ corresponding to the space covered by their two moving boundaries at time $t$. The second term corresponds to the domains with one collision, and the third term corresponds to the static distribution of domains with both sides blocked. This last term also corresponds to the final size PDF when $t \rightarrow \infty$, which is the Gamma probability function of equation (3) for $D=1$. The evolution of the total size PDF is depicted in figure 5, the final Gamma probability function is shown with a dashed line for reference. The emmergence of the final Gamma distribution as the transformation advances and more and more domains become completely blocked will be further discussed below.

\section{RESULTS FOR $\mathrm{D}=3$}

In a three dimensional PV transformation a collision configuration $\left\{l_{i}, \theta_{i}, \varphi_{i}\right\}(i=1 \ldots k)$ determines unequivocally any geometrical property of the domain. Likewise the previous development for the domain size in a $D=1$ space, we can define a function $Y_{k}\left(\left\{l_{i}, \theta_{i}, \varphi_{i}\right\}\right)$ which calculates a given geometrical property $y$ for a domain with collision configuration $\left\{l_{i}, \theta_{i}, \varphi_{i}\right\}$. Then, the time-invariant PDF of the $y$ property for the domains with a certain 
value of $k$ can be calculated as

$g_{k}^{Y}(y) d y=k !\left(\frac{3}{4 \pi}\right)^{k} \int_{l_{1}=0}^{1} \int_{\theta_{1}=0}^{\pi} \int_{\varphi_{1}=0}^{2 \pi} \ldots \int_{l_{k}=l_{k-1}}^{1} \int_{\theta_{k}=0}^{\pi} \int_{\varphi_{k}=0}^{2 \pi} \delta\left(y-Y_{k}\left(\left\{l_{i}, \theta_{i}, \varphi_{i}\right\}\right)\right) \prod_{i=1}^{k} l_{i}^{2} \sin \left(\theta_{i}\right) d l_{i} d \theta_{i} d \varphi_{i}$.

And now, the total PDF of the $y$ property at time $t$ can be obtained adding the contributions of the $k$-populations, this is

$$
g_{\text {total }}^{Y}(y, t)=\sum_{k=0}^{\infty} g_{k}^{Y}(y) T_{k}(t)
$$

The calculation of the $g_{k}^{Y}(y)$ functions is more or less complex depending on the geometrical property; in some cases the analytical form of the $g_{k}^{Y}(y)$ functions can be obtained [19], while in other cases a numerical integration of equation (22) is required. At low values of the transformed fraction $x(t)$, that is at the early stages of the transformation, the number of $k$ distributions required for the calculation of the total PDF is relatively low, but it increases as the transformation proceeds. Figure 6 shows the value of $k_{0.99}(t)$ which stands for the minimum number of $k$ needed to cover at least $99 \%$ of the total number of domains. As it is observed, the calculation of the total distribution of a certain geometric property at $t \rightarrow \infty$ using the present method becomes impractical because it may require the numerical calculation of an infinite number of $g_{k}^{Y}(y)$ functions. However, at any finite time $t$, the total PDF can be calculated to arbitrary accuracy adding a finite number of functions and, as it is observed in figure 6 , the number of $k$-populations needed for the calculation increases abruptly only at values of $x(t)$ very close to 1 when the overall geometrical configuration is practically static. This implies that even the final configuration at $x(t)=1$ can be approximated to any desired accuracy from the configuration obtained at $x(t) \lesssim 1$ using a finite number of $g_{k}^{Y}(y)$ functions.

\section{A. Temporal evolution of the free boundary distribution}

The free boundary fraction $b$ of a domain is now defined as the fraction of the original spherical boundary that is in contact with untransformed space, this is equal to the fraction of solid angle still not screened by collisions with neighbouring domains. In a PV transformation all the domains start growing simultaneously with equal growth velocity, then the collision between two domains can be interpreted as the intersection of two equally sized 
spheres of normalized radius 1 at distance $l_{i}$ from their centres. This intersection occupies a fraction $\frac{\left(1-l_{i}\right)}{2}$ of solid angle; however, in order to compute the free solid angle remaining after $k$ extended collisions at positions $\left\{l_{i}, \theta_{i}, \varphi_{i}\right\}(i=1 \ldots k)$, the overlaps between different collisions should be taken into account. In order to do this computation we define the function

$$
C_{i}\left(\theta, \varphi, l_{i}, \theta_{i}, \varphi_{i}\right)=H\left(d^{2}-1\right)
$$

with

$$
d^{2}=\left(\sin \theta \cos \varphi-2 l_{i} \sin \theta_{i} \cos \varphi_{i}\right)^{2}+\left(\sin \theta \sin \varphi-2 l_{i} \sin \theta_{i} \sin \varphi_{i}\right)^{2}+\left(\cos \theta-2 l_{i} \cos \theta_{i}\right)^{2}
$$

where $d$ is the distance between a boundary point $(1, \theta, \varphi)$ and the neighbouring nucleation point $O_{i}=\left(2 l_{i}, \theta_{i}, \varphi_{i}\right)$. This bivaluated function gives a value of 1 for any solid angle not occupied by the $i$-th extended collision, that is with $d>1$, and a value of 0 otherwise. With the aid of $C_{i}$, the function $B_{k}$ which gives the free boundary fraction for a given collision configuration can be written as

$$
B_{k}\left(l_{1}, \theta_{1}, \varphi_{1} \ldots, l_{k}, \theta_{k}, \varphi_{k}\right)=\frac{1}{4 \pi} \int_{\theta=0}^{\pi} \int_{\varphi=0}^{2 \pi} \prod_{i=1}^{k} C_{i}\left(\theta, \varphi, l_{i}, \theta_{i}, \varphi_{i}\right) \sin (\theta) d \theta d \varphi
$$

For $k=1$, the function $B_{1}\left(l_{1}, \theta_{1}, \varphi_{1}\right)$ reads

$$
B_{1}\left(l_{1}, \theta_{1}, \varphi_{1}\right)=\frac{1+l_{1}}{2} .
$$

With the definition of the $B_{k}$ functions the integration of equation (22) gives the timeinvariant free boundary probability density functions $g_{k}^{B}(b)$. Figure 7 shows the result of this integration for different values of $k$ ranging from 1 to 20 . The function corresponding to $k=0$ is obviously

$$
g_{0}^{B}(b)=\delta(b-1)
$$

for $k=1$ the integration of equation (22) reduces to a variable change $b=\frac{1+l_{1}}{2}$ giving

$$
g_{1}^{B}(b)=6(2 b-1)^{2} H\left(b-\frac{1}{2}\right)
$$

and the $g_{k}^{B}(b)$ functions with $k>1$ have been calculated by Monte Carlo numerical integration ensuring a relative error lower than $10^{-3}$. The mean value of each time invariant $g_{k}^{B}(b)$ function, this is the mean occupied solid angle in a domain with $k$ extended collisions, 
must be also time invariant and can be independently calculated[19]. Because of the random distribution of nucleation points, all the angular positions of the neighbouring domains

are equiprobable. Bearing in mind that each collision occupies a fraction $\frac{\left(1-l_{i}\right)}{2}$ of the free boundary, the average free boundary fraction of a domain with a set of collision times $\left\{l_{i}\right\}$ is

$$
\bar{b}_{k}\left(\left\{l_{i}\right\}\right)=\prod_{i=1}^{k} \frac{\left(1+l_{i}\right)}{2}
$$

and the mean value of $b$ for the population of domains with $k$ extended collisions is obtained to be

$$
\bar{b}_{k}=\int_{0}^{1} b g_{k}^{B}(b) d b=k ! \int_{l_{1}=0}^{1} \ldots \int_{l_{k}=l_{k-1}}^{1} \bar{b}_{k}\left(\left\{l_{i}\right\}\right) \prod_{i=1}^{k} l_{i}^{2} d l_{i}=\left(\frac{8-1}{8}\right)^{k} .
$$

The mean values $\bar{b}_{k}$ of the normalized probability functions are indicated in figure 7 .

Recalling equation (31), it confirms the result obtained in reference [18] that the mean free boundary of domains after $k$ extended collisions is

$$
\bar{b}_{k}=\left(\frac{2^{D}-1}{2^{D}}\right)^{k}
$$

which phisically means that, on average, the free boundary decreases by a factor $2^{-D}$ after each extended collision. Given that the number of orthogonal directions in a $D$-dimensional space is $2 D$ and the number of sectors divided by these orthogonal directions is $2^{D}$, it appears that an average of one sector (one quadrant for $D=2$ or one octant for $D=3$ ) is occluded by each extended collision, and not an orthogonal growth direction as it may intuitively appear.

Now, from equation $(23)$, the total PDF $g_{\text {total }}^{B}(b, t)$ is calculated adding the corresponding contributions of the $k$ domains at time $t$. Figure 8 shows the evolution of $g_{\text {total }}^{B}(b, t)$ at three different stages during the transformation. As all the domains have $b=0$ when $x(t) \rightarrow 1$, the function $g_{\text {total }}^{B}(b, t)$ tends to a Delta function $\delta(b-0)$ at the final stages of the transformation. In the calculations presented in figure 8, the total PDF was obtained using the contributions of the $g_{k}^{B}(b)$ functions with $k$ ranging from 0 to 20 . At $x(t)=0.75$ the domains with $k \leq 20$ represent more than $99 \%$ of the total. 


\section{B. Temporal evolution of the size distribution}

The calculation of the size PDF in $D=3$ is performed following the same steps than the free boundary fraction development of the previous section. First of all we define the normalized size of a domain

$$
s=\frac{3 a}{4 \pi t^{3}}
$$

where $a$ is the volume occupied by the domain at time $t$. Using the function $C_{i}$ defined in equations (24) and (25) the calculation of the size of a domain with a certain collision configuration can be obtained by

$$
S_{k}\left(l_{1}, \theta_{1}, \varphi_{1} \ldots, l_{k}, \theta_{k}, \varphi_{k}\right)=\frac{3}{4 \pi} \int_{r=0}^{1} \int_{\theta=0}^{\pi} \int_{\varphi=0}^{2 \pi} \prod_{i=1}^{k} C_{i}\left(\theta, \varphi, \frac{l_{i}}{r}, \theta_{i}, \varphi_{i}\right) r^{2} \sin (\theta) d r d \theta d \varphi,
$$

noting that $C_{i}\left(\theta, \varphi, \frac{l_{i}}{r}, \theta_{i}, \varphi_{i}\right)$ is equal to 1 for any point $(r, \theta, \varphi)$ nearer to the nucleation point of the domain than to the neighbouring nucleation point $O_{i}=\left(2 l_{i}, \theta_{i}, \varphi_{i}\right)$. Now, the integration of equation (22) gives the time invariant functions $g_{k}^{S}(s)$ depicted in figure 9 . The probability density function corresponding to $k=0$ is again a delta function

$$
g_{0}^{S}(s)=\delta(s-1),
$$

and the integration of equation (22) for $k=1$ is equivalent to the variable change

$$
s=S_{1}\left(l_{1}, \theta_{1}, \varphi_{1}\right)=\frac{1}{2}\left(1+\frac{l_{1}}{2}\left(3-l_{1}^{2}\right)\right)
$$

this leading to

$$
g_{1}^{S}(s)=\left(\frac{2 \sin \left[\frac{\arccos (2 s-1)+\pi}{3}\right]}{\sqrt{s-s^{2}}}-4\right) H\left(s-\frac{1}{2}\right) .
$$

As in the free boundary case, the $g_{k}^{S}(s)$ functions with $k>1$ have been computed by numerical integration of equation (22).

Similarly to the average free-boundary fraction in the previous section, the mean normalized size of the domains with a given number $k$ of extended collisions can be explicitly obtained giving

$$
\bar{s}_{k}=\int_{0}^{1} s g_{k}^{S}(s) d s=\frac{8}{k+1}\left(1-\frac{7^{k+1}}{8^{k+1}}\right) .
$$


This result is derived in appendix B and it can be extended to any dimension $D$ giving a value of

$$
\bar{s}_{k}=\frac{2^{D}}{k+1}\left[1-\left(\frac{2^{D}-1}{2^{D}}\right)^{k+1}\right] .
$$

The values of $\bar{s}_{k}$ corresponding to the plotted $g_{k}^{S}(s)$ functions are also shown in figure 9 . All the $g_{k}^{S}(s)$ functions obtained by numerical integration of equation (22) satisfy this mean value, this result ratifying the correctness of the computation.

Finally, the overall PDF $g_{\text {total }}^{S}(s, t)$ is calculated from equation (23) and the total PDF in terms of the volume $a$ instead of the normalized size $s$ can be obtained using the variable change in equation (33), this is

$$
f_{D=3}(a, t)=g_{\text {total }}^{S}(s, t) \frac{d s}{d a} .
$$

The results obtained for $f_{D=3}(a, t)$ at $x(t)=0.25,0.5$ and 0.75 are presented in figure 10 . As in the one dimensional case, the final static Gamma distribution is progressively obtained as more and more domains become completely blocked during the transformation. Similarly to the one and two dimensional cases [19], the analytical results obtained in this work have been checked against stochastic simulations of the system checking the correctness of the calculation. Details of these stochastic simulations can be found in references [6] and [16].

\section{ORIGIN OF THE FINAL GAMMA SIZE DISTRIBUTION}

The emmergence of a static Gamma distribution of sizes at the end of a PV transformation is not a surprising fact, the Gamma distribution is linked to Poisson processes by definition. Considering a Poisson process with rate $\lambda$, the probability of the $h$-th Poisson event occurring at time $t$ is given by a Gamma distribution with probability density function

$$
p(h, t)=\frac{\lambda(\lambda t)^{h-1}}{(h-1) !} \exp (-\lambda t)
$$

which is equivalent to equation (3) with $\nu=h$ and a mean value $\rho^{-1}=\nu \lambda^{-1}$. Therefore, the final size distribution of a PV transformation might be considered as the probability of finding a domain with a certain set of collisions at distances (or times) distributed Poissonlike. This is straightforwardly seen for the one-dimensional system, where each domain grows from its nucleation point in two branches occupying respectively the left and the right hand side untransformed spaces. The growth of these branches is stopped after one 
collision at distance $t$ with a neighbouring domain, this neighbour having a nucleation point at distance $2 t$. Hence, such collisions have a Poisson probability exp $(-2 \rho t)$ with rate $\lambda=2 \rho$ of occurring at time $t$ and the probability of having the first collision in one of the branches at time $t$ is given by

$$
p(1, t)=2 \rho \exp (-2 \rho t) .
$$

Then, the probability of having a domain branch with final length $a_{j}=t$ is given by

$$
f_{\text {branch }}\left(a_{j}\right)=2 \rho \exp \left(-2 \rho a_{j}\right)
$$

and the probability of having a domain (formed by two branches) with final size $a=a_{1}+a_{2}$ is given by the convolution of two of these functions

$$
f_{\text {domain }}(a)=\int_{0}^{\infty} \int_{0}^{\infty} f_{\text {branch }}\left(a_{1}\right) f_{\text {branch }}\left(a_{2}\right) \delta\left(a_{1}+a_{2}-a\right)=4 \rho^{2} a \exp (-2 \rho a)
$$

which is the Gamma probability function with $\nu=2$ corresponding to a one-dimensional PV tessellation.

For $D=2$ and $D=3$ systems a rude similar approach can be attempted. Let us consider the growth of a domain as the growth of $n$ branches each one occupying a fraction of angle $(D=2)$ or solid angle $(D=3)$, the growth of each branch will stop after colliding once in the corresponding sector. In order to follow with this approach we assume each branch occupying an equal sector $\frac{D K_{D}}{n}\left(\frac{2 \pi}{n}\right.$ for $D=2$ and $\frac{4 \pi}{n}$ for $\left.D=3\right)$. In each one of these sectors, the probability of having the first collision with a neighbouring nuclei at time $t$ is given by

$$
\frac{D K_{D} 2^{D} t^{D-1} \rho}{n} \exp \left(-\frac{K_{D} 2^{D} t^{D} \rho}{n}\right)
$$

which is the probability of finding a neighbouring nucleation point at distance $2 t$ inside the sector, multiplied by the probability of finding no other nucleus at smaller distance. Hence, the probability function of having a branch occupying an area $a_{j}=\frac{\pi t^{2}}{n}$ for $D=2$ or a volume $a_{j}=\frac{4 \pi t^{3}}{3 n}$ for $D=3$ is given by

$$
f_{\text {branch }}\left(a_{j}\right)=2^{D} \rho \exp \left(-2^{D} \rho a_{j}\right)
$$

and the probability of a domain to have a size $a=a_{1}+\ldots+a_{n}$ is obtained if we convolute $n$ of these probability functions obtaining

$$
f_{\text {domain }}(a)=\frac{\left(2^{D} \rho\right)^{n} a^{n-1}}{(n-1) !} \exp \left(-2^{D} \rho a\right)
$$


Restricting the possible size probability functions to functions with expected value of $\rho^{-1}$ we have that only $n=4$ and $n=8$ for $D=2$ and $D=3$ are allowed, in which case the two distributions become Gamma probability functions with $\nu=2^{D}$. It should be noted that this approach is inspired in ref. [15]. Here, however, the probability functions are constructed in function of the occupied space in each sector instead of the collision distance, this allows a complete analytical treatment and extension to the $D=3$ case.

The actual size distribution of a PV tessellation have values of the exponent $\nu$ different from $2^{D}$. Figure 11 shows the comparison between the actual final Gamma size probability functions in PV transformations in $D=2$ and $D=3$ spaces and the Gamma functions with $\nu=4$ and $\nu=8$ derived from this approach, it is observed that the distributions with exponent $2^{D}$ have smaller dispersion around the mean value. From the approach presented here, the value of the exponent is related to the average number of first neighbours of a domain. In a PV tessellation the average number of real collisions is 6 and 15.54 for $D=2$ and $D=3$ respectively[7], which are very different from the $2^{D}$ collisions considered in the approach. However, the average number of 'full neighbours', defined as the number of neighbouring nucleation points that can be connected with the domain origin with a straight line without crossing a third domain, is indeed $2^{D}[7]$. Then, the structure can be interpreted as domains with an average of $2^{D}$ main collisions but with a large geometric anisotropy produced by secondary collisions in the remaining solid angle fraction not obstructed by full neighbours. Contrary to the $D=1$ system, where all domains have exactly 2 real collisions, for $D>1$ the number of possible real collisions of a completely blocked domains goes from 3 $(D=2)$ or $4(D=3)$ to infinity, implying a wide distribution of probable domain geometries. This wider distribution of domain shapes produces a larger size dispersion around the mean value, which implies a smaller exponent in the Gamma distribution as its variance is given by $\nu^{-1} \rho^{-2}$.

From this approach it may be argued that the presence of Gamma size distributions in physical systems must have its origin in a random distribution of nucleation points or initial particle positions. In different systems, experimental distributions of area or volume are fitted by statistical distributions such as the Gauss, Gamma and log-normal[20]. If the structure is uniquely determined by a Poisson point process the Gamma distribution appears, this is the case in different types of phase transformations where the final structure is completely dependent on the initial random distribution of seeds [21][22]. When the structure is allowed 
to relax under some particular dynamics the anisotropy of the domains is generally reduced, this leading to different types of domain size distributions. In coarsening structures driven by surface tension, the unfavourable smallest domains disappear, the structure coarsens but an invariant distribution of sizes appears. In many cases these structures produce an inverse exponential size distribution with larger size dispersion than the Gamma distribution [23][24], this larger dispersion is originated by the continuously growth and shrinkage of the favourable and unfavourable cells although the shape of the domains is much more uniform than in a PV tessellation. These microstructures are typically observed during normal grain growth in metallurgy[25] and soap bubble froths[24].

In other cases, after some degree of relaxation the system continues to present a Gamma distribution of sizes but with a more uniform distribution of cell shapes and sizes than in a PV tessellation. This seems to be the case in sphere packings and some other granular materials[26] [27]. In ref. [26] the topological parameters of the Voronoi cells found in uniform sphere packing simulations with low density are very close to the values of a PV tessellation. They found that the number of faces per cell $N F$ and the surface cell area $S C$ increase when reducing packing density $P D$. For high packing densities, $P D=0.605$, they found $N F=14.41$ and $S C=5.45 \bar{a}^{2 / 3}$ ( $\bar{a}$ being the mean cell volume), while at $P D=0.188$ they found $N F=15.33$ and $S C=5.87 \bar{a}^{2 / 3}$. It should be noted that values of $N F=15.54$ and $S C=5.82 \bar{a}^{2 / 3}$ correspond to a PV tessellation while $N F=14$ and $S C=5.32 \bar{a}^{2 / 3}$ correspond to an ordered cube-octahedron structure[7]. Therefore, the structure changes from being composed by highly asymmetric cells with a large variance of sizes at low packing densities to a more uniform and narrower Gaussian-like distribution at high packing densities. In ref. [27] they obtained Gamma-like size distributions for the cell volumes of sphere packings at all densities, these distributions have an exponent $\nu$ that decreases towards the value corresponding to a PV tessellation $(\nu=5.586)$ for low packing densities. Following the present approach, the presence of Gamma-like size distributions in these systems may be interpreted as a signature of the initial completely random distribution of sphere positions, the corresponding Voronoi cell structure becoming more uniform (this means larger $\nu$ values) as the sphere positions are accomodated to higher packing densities. An interesting point in ref. [27] is the reduction of the exponent of the Gamma distributions when the increase in density causes a transition towards a jammed state. From a pure topological view, this reduction of the exponent may be due to the continous relaxation of some parts 
of the structure towards a more uniform and dense distribution of local environments while some other parts are arrested in lower density local configurations, this would increase the size dispersion around the mean value implying a reduction of the exponent.

However, the validity of the gamma distribution and the values of the exponent $\nu$ for $D=2$ and $D=3 \mathrm{PV}$ structures remain as a semi-empirical result. In this issue the comparison with the completely solvable $D=1$ case may give us some clues. In sections III and IV we obtained that the Gamma probability function appears adding the contributions of $g_{k}^{S}(s)$ functions corresponding to totally blocked domains, this means domains with $s<1 / 2$ in the $D=1$ case. Inspecting equation (19) we observe that these probability functions of normalized sizes are Beta probability functions

$$
\frac{\Gamma(\alpha+\beta) s^{\alpha-1}(1-s)^{\beta-1}}{\Gamma(\alpha) \Gamma(\beta)}
$$

with parameters $\alpha=\nu$ and $\beta=k-1$. For large $k$ numbers the contribution of partially blocked domains, this means the $s>1 / 2$ part of the $g_{k}(s)$ functions, tends to disappear they becoming pure Beta probability functions. For $D=2$ and $D=3$ the same behaviour is found for the $g_{k}^{S}(s)$ functions. Figure 12 shows the $g_{k}^{S}(s)$ functions computed numerically from equations (22) and (34) compared with Beta probability functions with parameters

$$
\alpha=\nu, \quad \beta(k)=\frac{(k+1) \nu}{2^{D}\left[1-\left(\frac{2^{D}-1}{2^{D}}\right)^{k+1}\right]}-\nu .
$$

The value of parameter $\beta(k)$ is introduced in order to obtain Beta probability functions with mean value equal to the value of $\bar{s}_{k}$ calculated in the previous section. It is observed that the agreement between the calculated functions and the Beta functions is very good for large $k$ numbers, in which the $g_{k}^{S}(s)$ functions are expected to describe the size distribution of mainly completely blocked domains. However, it should be noted that the domain populations with $k=10(D=2)$ and $k=20(D=3)$ extended collisions, which are the larger $k$ numbers shown in the figure, still have a significant proportion of non-blocked domains. Therefore, it is expected that the domains with $k \gg 1$ will have domain size distributions very well described by a Beta distribution. Furthermore, for $k \gg 1$ parameter $\beta(k)$ can be reduced to $\beta(k)=\left(\frac{(k+1)}{2^{D}}-1\right) \nu$, giving the value of $\beta=k-1$ found for the completely blocked domains in $D=1$.

Now, we propose the normalized size distributions of completely blocked domains with $k$ 
extended collisions to be Beta-like distributions that can be written as

$$
g_{k}^{\text {Blocked }}(s)=\frac{\Gamma\left(\nu \frac{(k+1)}{2^{D}}\right) s^{\nu-1}(1-s)^{\nu \frac{(k+1)}{2^{D}}-\nu-1}}{\Gamma(\nu) \Gamma\left(\nu \frac{(k+1)}{2^{D}}-\nu\right)}
$$

and then the Gamma probability function appears as

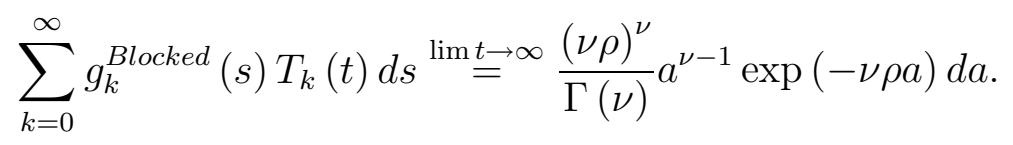

which is the sum of Beta probability functions weighted by the relative number of $k$-domains at time $t$. Equation (51) can be easily proofed if $\nu=2^{D}$, but it is found valid for any value of $\nu$ considering a sufficiently large time $t$. It should be noted that at $t \rightarrow \infty$, that is when the Gamma distribution is formed at the end of the transformation, only domains with $k \gg 1$ have significant contributions to the total.

Similarly to the Gamma size distribution, the validity of Beta distributions of normalized sizes for domains with $k$ extended collisions is at present a heuristic result. The calculation, by means of the method presented in this article, of the distribution of sizes for completely blocked domains as well as the distributions of other geometrical parameters such as the number of faces, edges, or "full neighbours" may cast light into the origin of the dispersion of sizes and shapes arising from the random distribution of points and the value of the exponent $\nu$ in the Gamma and Beta distributions discussed in this section. Such calculations are currently in progress.

\section{CONCLUSIONS}

Poisson-Voronoi (PV) space tessellations and transformations are found in many different systems concerning biology[28], geology [14], chemistry[29][30], metallurgy[31] and others. In many cases the presence of a PV structure is related to a young or initial cellular structure [24], which subsequently evolves or relaxes to a more stable structure following the particular dynamics of the system. The analytical knowledge of the initial distribution of sizes or other geometric characteristics of the domains, such as the number of vertices and edges or the boundary area, may be of great interest for the mathematical modelling of these systems.

In this article, the geometric configuration generated during a PV transformation of

arbitrary dimensionality has been described in terms of populations of domains with a 
given number $k$ of extended collisions. It has been found that for these $k$-populations the probability distribution of any geometric property is time invariant; only their relative amount changes along the growth of the structure with a well defined probability $T_{k}(t)$. This can be applied to compute the temporal evolution of the overall probability density function of any geometric property of the domain structure. This development, that was previously applied to a two-dimensional transformation, has been extended and generalized here to one and three dimensions. In the one-dimensional case, the development gives an explicit solution and the results previously obtained by Schulze [17] are reproduced. In the three-dimensional case, the temporal evolution of the domain size and free boundary distributions can be analytically calculated for the first time and general expressions for extending the calculation to other domain characteristics have been presented.

The results presented here give a deep and accurate knowledge of the geometric structure during a PV transformation; this enables the study of properties and spatial correlations in partially occupied systems originated in nucleation and growth processes. Moreover, the application of the present general method to the $D=1$ case has allowed us to perform a direct comparison between the explicit expressions obtained for $D=1$ and the numerical results computed for $D>1$. This gives some hints to the explicit form of the probability functions obtained for $D>1$ where the infinite number of growth directions prevents, till this moment, to derive explicit results. As shown in section $\mathrm{V}$, if explicit general expressions of the time invariant size distributions could be obtained for any $D$, this may lead to the theoretical proof of the Gamma distribution of sizes observed in PV tessellations.

Finally, the validity of the KJMA equation has been proofed for a wide range of processes with different nucleation and growth laws[9] [32]. Therefore, although the application of the calculation method is restricted to a PV transformation, the fact that the KJMA equation is obtained here as a result of the time invariant geometric distributions [18] suggests that a clever normalization of the domains may allow the extension of the method to transformations with more complex nucleation and growth laws.

\section{Acknowledgments}

This work was funded by CICYT, Grant No. MAT2007-60087, and Generalitat de Catalunya, Grant No. 2005SGR00535. The authors thank Dr. Monica Blanco and Dr. 
Marta Ginovart for many fruitful discussions and valuable help.

\section{Appendix A:}

Integration of equation (18) is clearly deduced considering certain $k$ numbers. Let us first consider $k=1$, in such case the integration reduces to the variable change

$$
g_{1}(s) d s=\int_{l_{1}=0}^{1}\left[\delta\left(s-\frac{1+l_{1}}{2}\right)\right] d l_{1}=2 H\left(s-\frac{1}{2}\right)
$$

where the fact that $0<l_{1}<1$ and then $\frac{1}{2}<s<1$ leads to the heaviside step function. Now, considering $k=2$ we have

$$
g_{2}(s) d s=2 \int_{l_{1}=0}^{1} \int_{l_{2}=l_{1}}^{1}\left[\left(\frac{1}{2}\right) \delta\left(s-\frac{1+l_{1}}{2}\right)+\left(\frac{1}{2}\right) \delta\left(s-\frac{l_{1}+l_{2}}{2}\right)\right] d l_{2} d l_{1}
$$

the integration of the two terms inside the brakets, taking into account the integration limits of $l_{1}$ and $l_{2}$, results in

$$
\begin{aligned}
g_{2}(s) d s & =\left[4(1-s) H\left(s-\frac{1}{2}\right)\right]+\left[2(1-s) H\left(s-\frac{1}{2}\right)+2 s H\left(\frac{1}{2}-s\right)\right]= \\
& =6(1-s) H\left(s-\frac{1}{2}\right)+2 s H\left(\frac{1}{2}-s\right) .
\end{aligned}
$$

Proceeding likewise for larger $k$ numbers we obtain

$$
\begin{gathered}
g_{3}(s) d s=12(1-s)^{2} H\left(s-\frac{1}{2}\right)+6 s(1-s) H\left(\frac{1}{2}-s\right) \\
g_{4}(s) d s=20(1-s)^{3} H\left(s-\frac{1}{2}\right)+12 s(1-s)^{2} H\left(\frac{1}{2}-s\right) \\
\vdots \\
g_{k}(s) d s=k(k+1)(1-s)^{k-1} H\left(s-\frac{1}{2}\right)+k(k-1) s(1-s)^{k-2} H\left(\frac{1}{2}-s\right)
\end{gathered}
$$

which is the result in equation (19).

\section{Appendix B:}

In order to calculate the mean normalized size of a domain with $k$ extended collisions let us consider a set of collision times $\left\{l_{i}\right\}(i=1 \ldots k)$ and a spherical shell at a normalized 
distance $r$ from the domain origin. The fraction of this shell not occupied by a particular collision at $l_{i}$ is

$$
c\left(l_{i}, r\right)=\left\{\begin{array}{c}
\frac{\left(1+l_{i} / r\right)}{2}, \quad \text { for } r>l_{i} \\
1, \quad \text { for } r<l_{i}
\end{array} .\right.
$$

Figure 13 shows a schematic view of a domain with a collision at $l_{i}$ and a domain shell (dashed line) at distance $r$. The fraction of domain shell not occupied by the $i$-th collision is $c=\frac{1-l_{i}}{2}$ at distance $r=1$ and $c=0$ at distance $r=l_{i}$.

As any angular position of the surrounding domains is equiprobable, then the average fraction of this shell not occupied by any of the $k$ extended collisions is given by

$$
\bar{b}_{k}\left(\left\{l_{i}\right\}, r\right)=\prod_{i=1}^{k} c\left(l_{i}, r\right)
$$

and the mean value over all the possible configurations $\left\{l_{i}\right\}$ is obtained to be

$$
\bar{b}_{k}(r)=k ! \int_{l_{1}=0}^{1} \ldots \int_{l_{k}=l_{k-1}}^{1} \bar{b}_{k}\left(\left\{l_{i}\right\}, r\right) \prod_{i=1}^{k} l_{i}^{2} d l_{i}=\left(\frac{8-r^{3}}{8}\right)^{k} .
$$

The derivation of this result can be more easily illustrated considering small $k$ values. For $k=1$ it is clear that

$$
\bar{b}_{1}(r)=\int_{l_{1}=0}^{r} \frac{\left(1+l_{1} / r\right)}{2} l_{1}^{2} d l_{1}+\int_{l_{1}=r}^{1} l_{1}^{2} d l_{1}=\frac{8-r^{3}}{8} .
$$

For $k=2$ the integration must be performed taking into account that $l_{1}<l_{2}$, this results in the following integration

$$
\begin{aligned}
\bar{b}_{2}(r) & =2 !\left[\int_{l_{1}=0}^{r}\left(\int_{l_{2}=l_{1}}^{r} \frac{\left(1+l_{1} / r\right)}{2} \frac{\left(1+l_{2} / r\right)}{2} l_{2}^{2} d l_{2}+\int_{l_{2}=r}^{1} \frac{\left(1+l_{1} / r\right)}{2} l_{2}^{2} d l_{2}\right) l_{1}^{2} d l_{1}+\int_{l_{1}=r}^{1} \int_{l_{2}=l_{1}}^{1} l_{2}^{2} l_{1}^{2} d l_{2} d l_{1}\right]= \\
& =\left(\frac{8-r^{3}}{8}\right)^{2} .
\end{aligned}
$$

The result in equation (B3) is obtained by induction proceeding likewise for larger $k$ values.

Therefore, the mean normalized size of a domain with $k$ extended collisions, that is the mean value $\bar{s}_{k}=\int_{0}^{1} s g_{k}^{S}(s) d s$ of the time invariant size probability density functions, can be calculated giving

$$
\bar{s}_{k}=4 \pi \int_{0}^{1} \bar{b}_{k}(r) r^{2} d r=\frac{8}{k+1}\left(1-\frac{7^{k+1}}{8^{k+1}}\right) .
$$


[1] D. Stoyan, W. S. Kendall and J. Mecke, Stochastic Geometry and Its Application (AkademieVerlag Berlin, Berlin, 1989), Chap. 10, p. 260.

[2] P. A. Mulheran, Philos. Mag. Lett. 66, 219 (1992).

[3] W. Christian, The Theory of Transformations in Metals and Alloys (Pergamon Press, Oxford, 1975), Chap. 12, p. 542.

[4] H. J. Frost and C. V. Thompson, Acta metall. 35, 529 (1987).

[5] W. S. Tong, J. M. Rickman and K. Barmak, Acta Mater. 48, 1181 (2000).

[6] E. Pineda and D. Crespo, Phys. Rev. B 60, 3104 (1999).

[7] J. L. Meijering, Philips Res. Rep. 8, 270 (1953).

[8] E. N. Gilbert, The Annals of Mathematical Statistics 33, 958 (1962).

[9] M. P. Shepilov and D. S. Baik, J. Non-Cryst. Solids 171, 141 (1994).

[10] M. Avrami, J. Chem. Phys. 7, 1103 (1939).

[11] W. A. Johnson and P. A. Mehl, Trans. Am. Inst. Mining and Metallurgical Engineers 135, 416 (1939).

[12] A. N. Kolmogorov, Bull. Acad. Sci. USSR, Phys. Ser. 1, 355 (1937).

[13] T. Kiang, Zeitschrift fur Astrophisik 64, 433 (1966).

[14] I. K. Crain, Computers \& Geosciences 4, 131-141 (1978).

[15] D. Weaire, J. P. Kermode and J. Weichert, Phil. Mag. B 53, L101 (1986).

[16] E. Pineda, P. Bruna and D. Crespo, Phys. Rev. E 70, 066119 (2004).

[17] G. E. W. Schulze, Journal of Crystal Growth 62, 7 (1983).

[18] E. Pineda, V. Garrido and D. Crespo, Phys. Rev. E 75, 040107(R) (2007).

[19] E. Pineda and D. Crespo, J. Stat. Mech.-Theory Exp., P06007 (2007).

[20] M. F. Vaz and M. A. Fortes, Scripta Metallurgica 22, 35 (1988).

[21] G. E. W. Schulze and L. O. Schwan, J. Mater. Sci. 28, 2706 (1993).

[22] P. Bruna, E. Pineda, D. Crespo and R. González-Cinca, Solid State Transformation and Heat Treatment (Edited by A. Hazotte, Wiley-VCH Verlag, Weinhein, 2005), p.126.

[23] P. A. Mulheran, Phys. Rev. E 51, R3803 (1995).

[24] N. Rivier, Phil. Mag. B 52, 795-819 (1985).

[25] M. Hillert, Acta Metallurgica 13, 227 (1965). 
[26] R. Y. Yang, R. P. Zou and A. B. Yu, Phys. Rev. E 65, 041302 (2002).

[27] T. Aste and T. Di Matteo, Phys. Rev. E 77, 021309 (2008).

[28] K. J. Dormer, Fundamental Tissue Geometry for Biologists (Cambridge University Press, 1980).

[29] A. Korobov, J. Math. Chem. 24, 261 (1998).

[30] W. S. Tong, J. M. Rickman, K. Barmak, J. Chem. Phys. 114, 915 (2001).

[31] B. N. Boots, Metallogr. 15, 53 (1982).

[32] V. Z. Belenkii, Geometriko-Veroyatnostnie Modeli Kristallizatsii/Geometrical-Probability Models of Crystallization (Nauka, Moscow, 1980). 
FIG. 1: Evolution of the transformed fraction and local spatial configuration at three different stages during a $D=1 \mathrm{PV}$ transformation.

FIG. 2: Evolution of the transformed fraction and local spatial configuration at three different stages during a $D=2 \mathrm{PV}$ transformation.

FIG. 3: (Color on-line). Time-invariant size probability density functions of domains with a number of extended collisions $k=1,2,3,4$ and 5 in a $D=1 \mathrm{PV}$ transformation.

FIG. 4: (Color on-line). Total domain-size distribution in a $D=1 \mathrm{PV}$ transformation at a transformed fraction $x(t)=0.6$. Calculated size distribution (thick line) compared with the results of a stochastic simulation (bars). The contribution of each of the $g_{k}(s)$ functions (with $k=0,1$, $2,3,4$ and 5) is also shown.

FIG. 5: Calculated domain-size probability density function at $x(t)=0.4,0.8$ and 1 in a $D=1$ PV transformation. The dashed line corresponds to the final gamma distribution.

FIG. 6: Number of $k$-populations needed to encompass $99 \%$ of the domains as a function of the overall transformed fraction.

FIG. 7: (Color on-line). Time-invariant free-boundary fraction probability density functions of domains with a number of extended collisions from $k=1$ to $k=20$ in a $D=3 \mathrm{PV}$ transformation. Dashed lines: Position of the mean value $\bar{b}_{k}$ of each distribution.

FIG. 8: Total free-boundary fraction probability density function in a $D=2 \mathrm{PV}$ transformation at three different transformed fractions $x(t)=0.05,0.25,0.5$ and 0.75 .

FIG. 9: (Color on-line). Time-invariant size probability density functions of domains with a number of extended collisions from $k=1$ to $k=20$ in a $D=3 \mathrm{PV}$ transformation. Dashed lines: Position of the mean value $\bar{s}_{k}$ of each distribution.

FIG. 10: Calculated size probability density function at $x(t)=0.25,0.5$ and 0.75 in a $D=3 \mathrm{PV}$ transformation. The dashed line corresponds to the final gamma distribution. 
FIG. 11: Comparison of Gamma size distributions. Two-dimensional system (Top): Distributions with exponent $\nu=3.575$ (dashed line) and $\nu=4$ (solid line). Three-dimensional system (Bottom): Distributions with exponent $\nu=5.586$ (dashed line) and $\nu=8$ (solid line).

FIG. 12: (Color on-line). Comparison of the proposed time-invariant Beta distributions of normalized sizes (solid lines) with the $g_{k}^{S}(s)$ functions obtained by numerical integration of equation (22) (symbols). Two-dimensional system (Top): $k=3$ (blue), $k=6$ (red), $k=8$ (green), $k=10$ (orange). Three-dimensional system (Bottom): $k=4$ (blue), $k=10$ (red), $k=15$ (green), $k=20$ (orange).

FIG. 13: Sketch of a domain with a collision at $l_{i}$. 


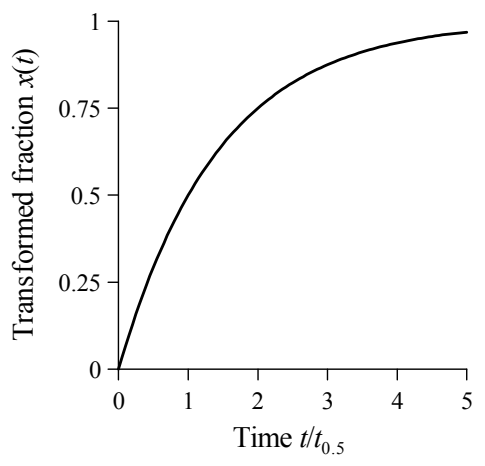

$x(t)=0.33$

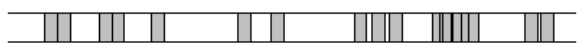

$x(t)=0.66$

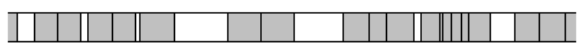

$x(t)=1$

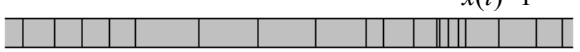

Figure 1 


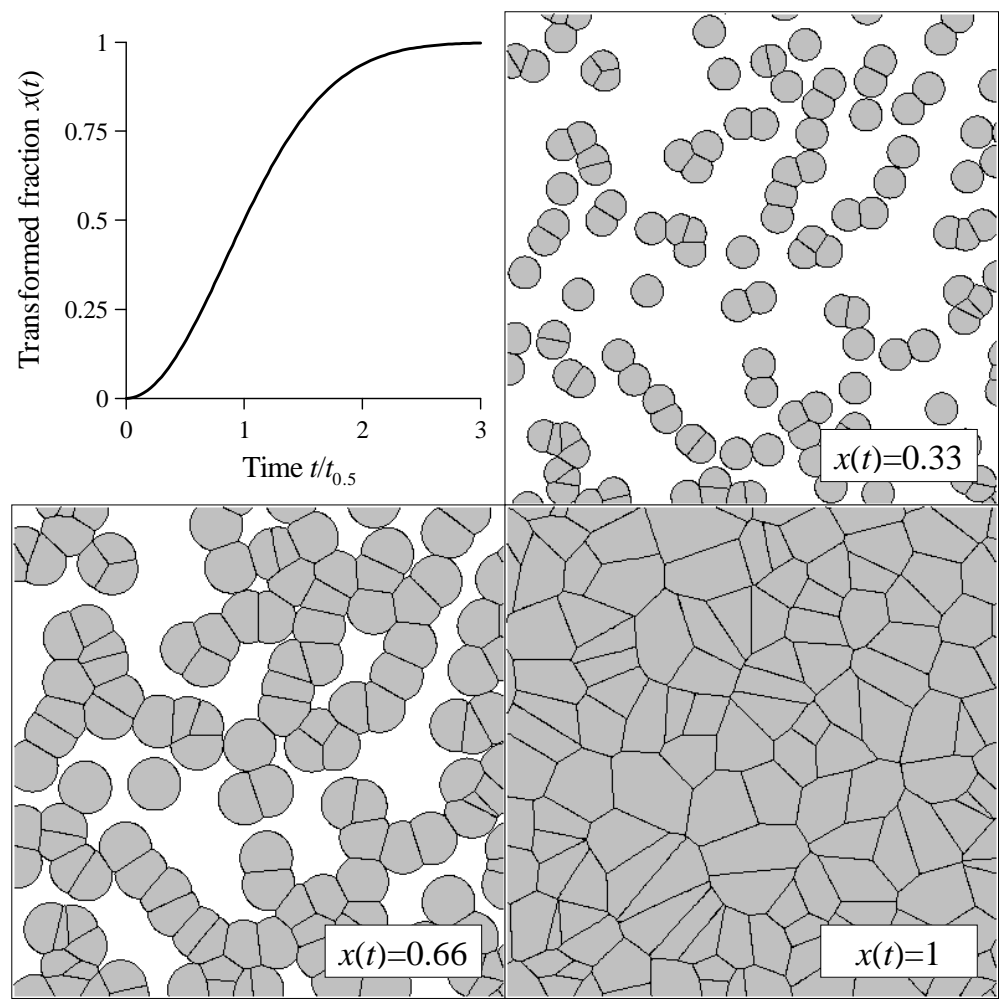

Figure 2 


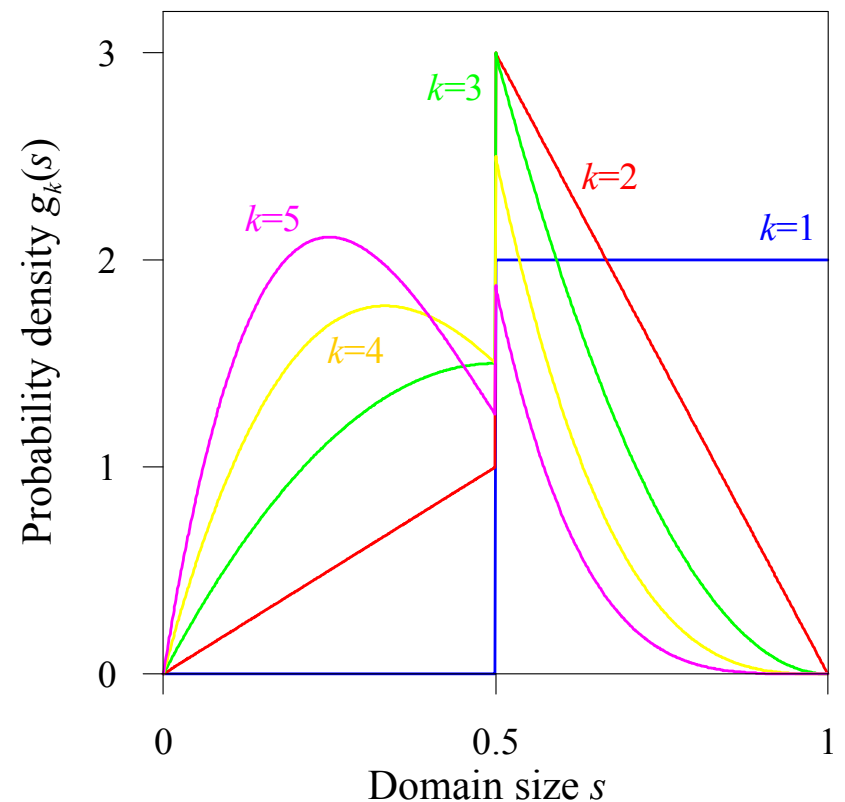

Figure 3.

Figure 3 


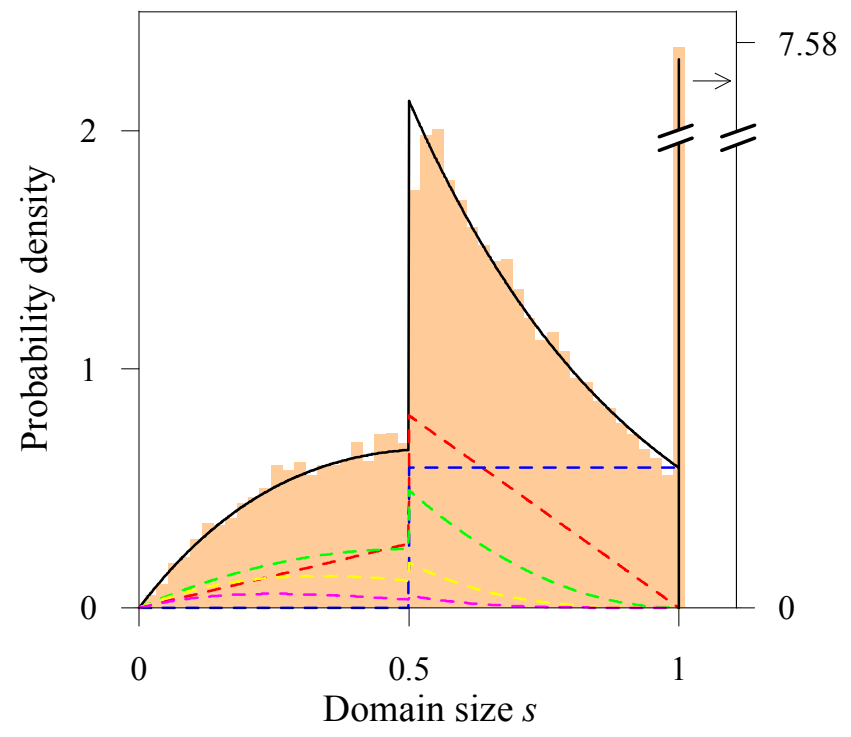

Figure 4.

Figure 4 


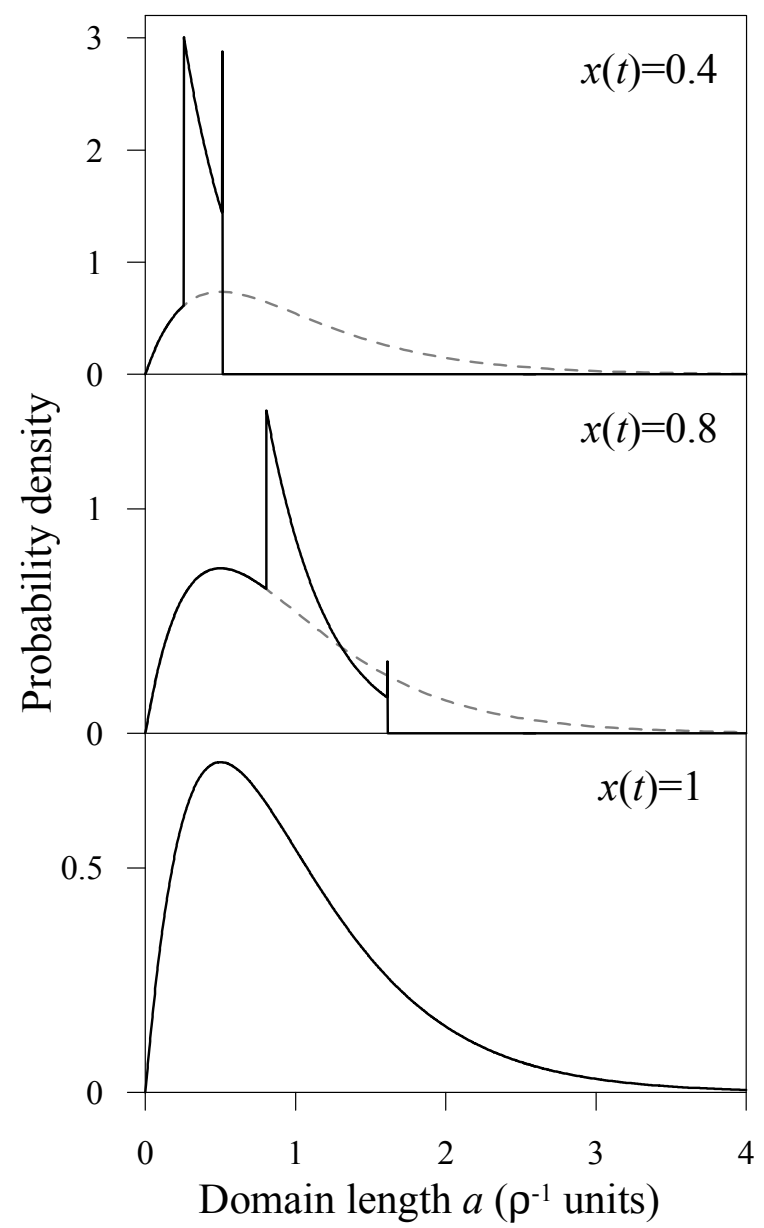

Figure 5.

Figure 5 


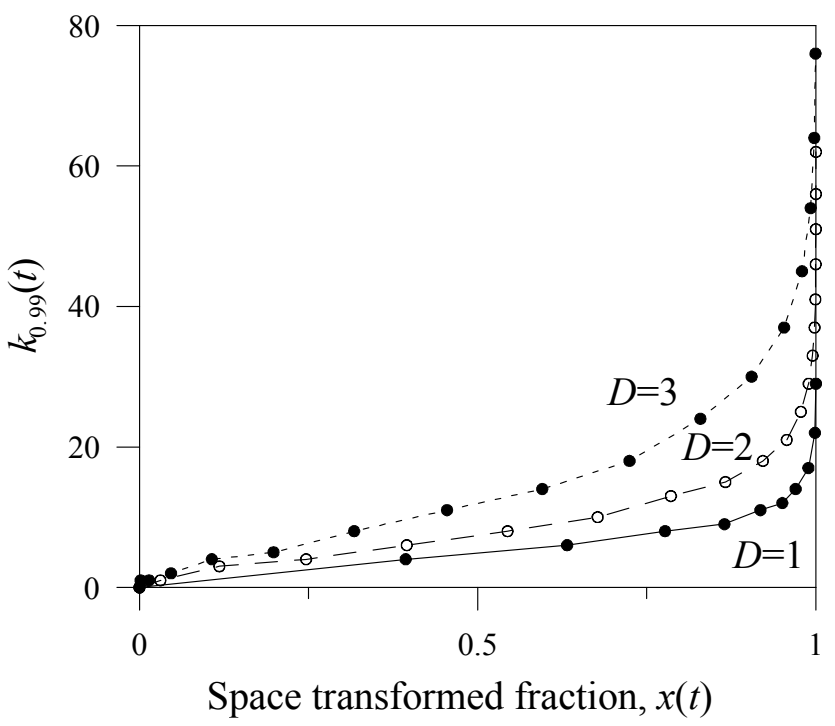

Figure 6. 


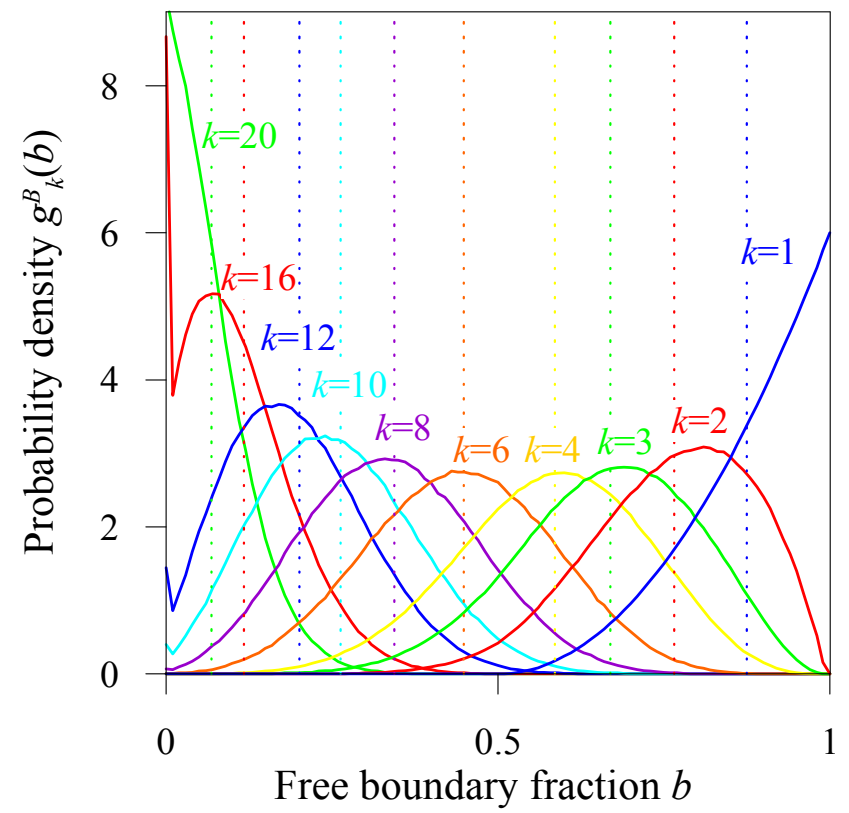

Figure 7.

Figure 7 


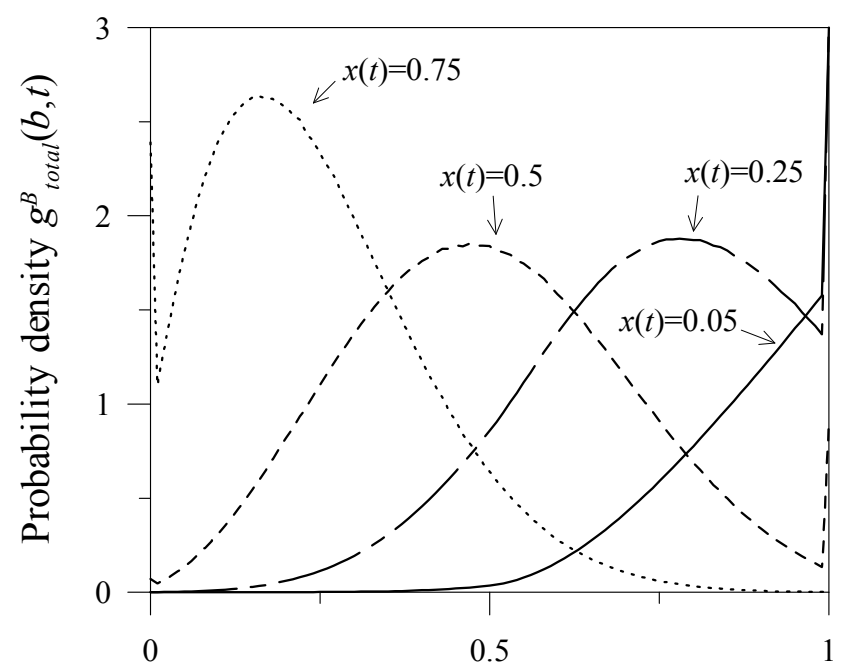

Free-boundary fraction $b$

Figure 8.

Figure 8 


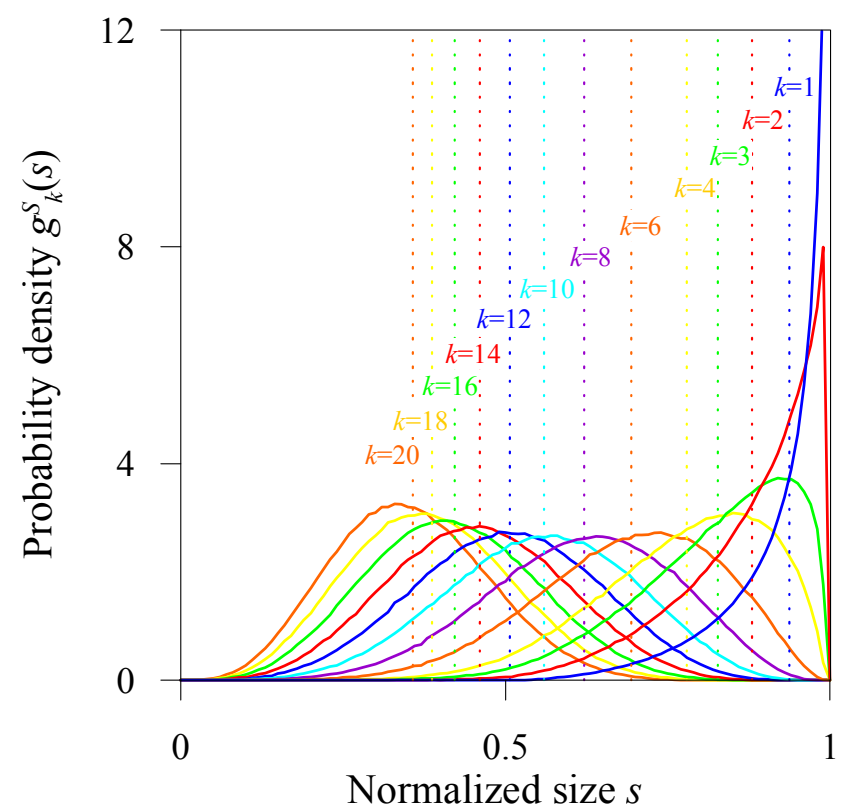

Figure 9.

Figure 9 


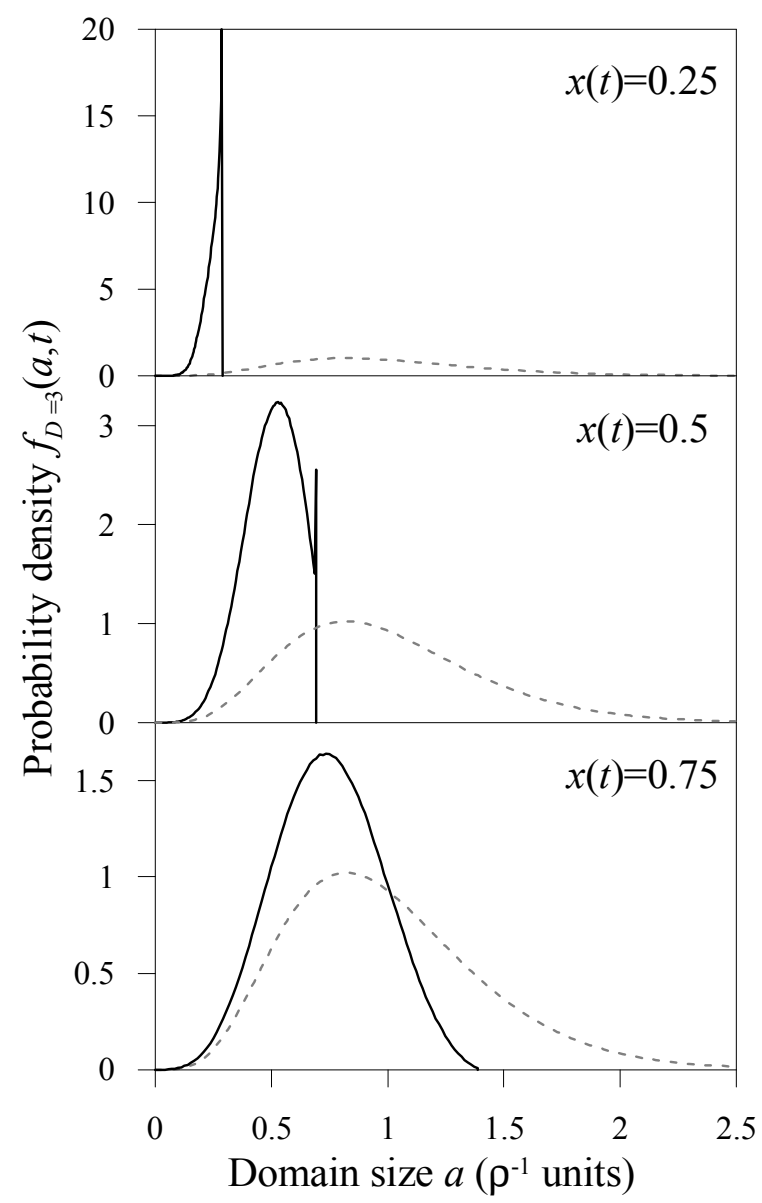

Figure 10.

Figure 10 


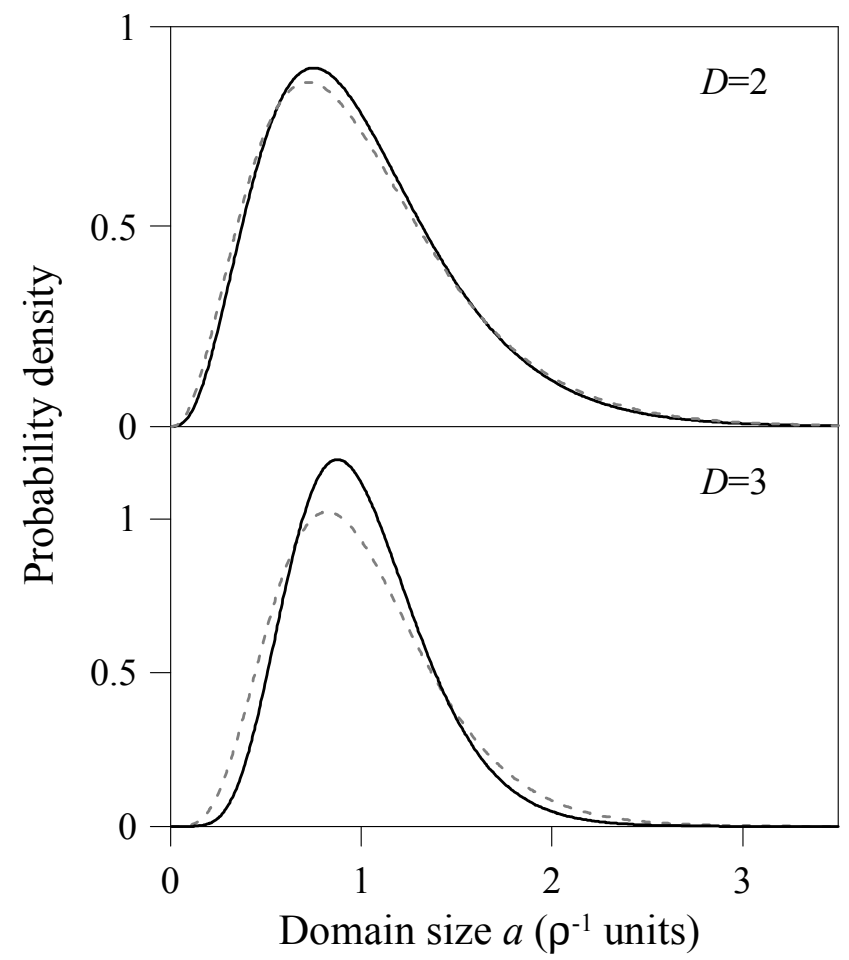

Figure 11.

Figure 11 


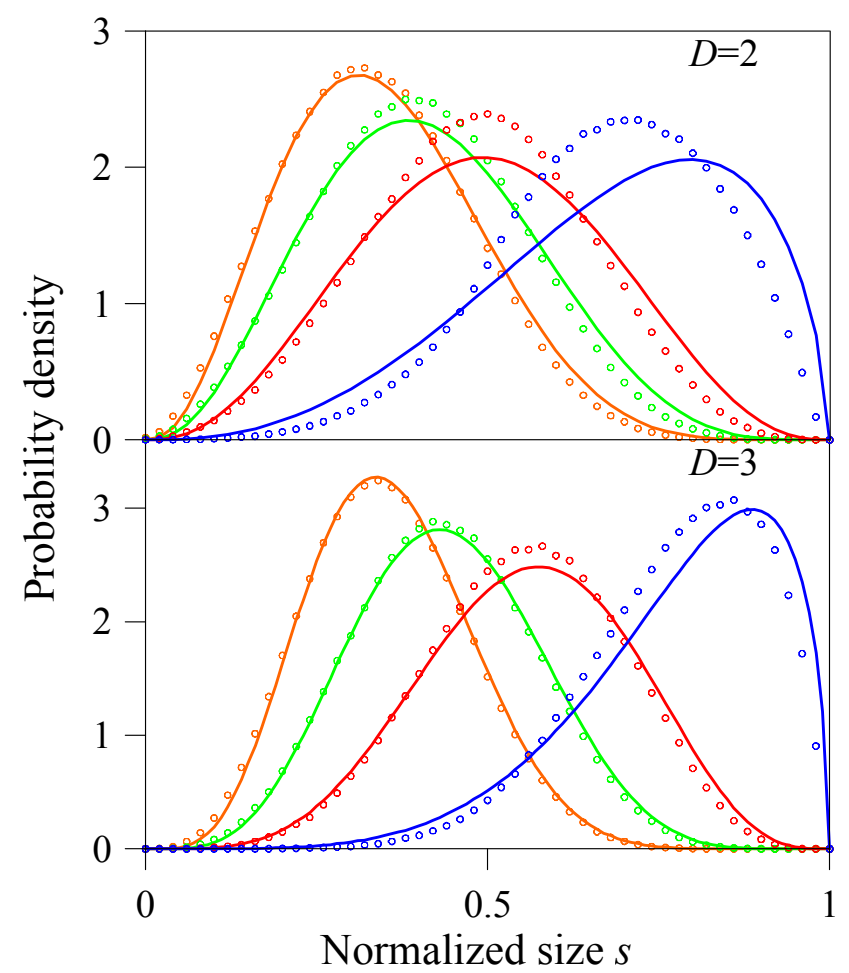

Figure 12.

Figure 12 


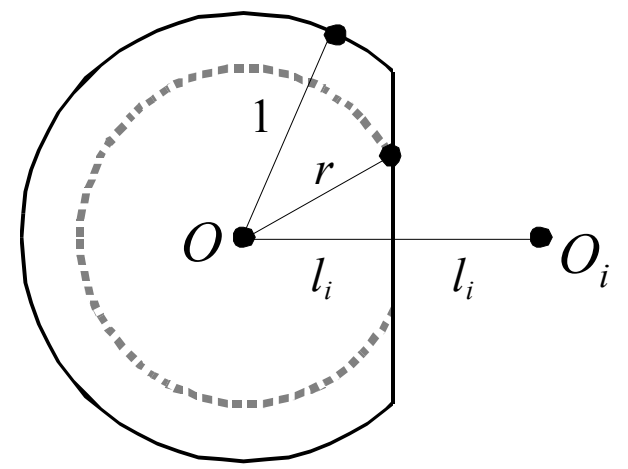

Figure Appendix B 1

Figure 13 